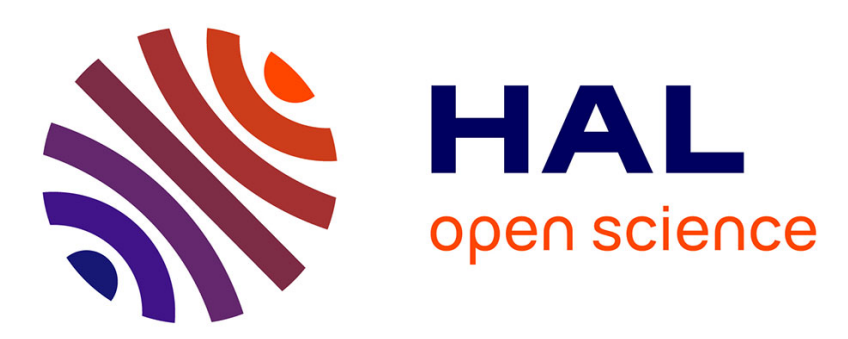

\title{
CW-pumped polarization-maintaining Brillouin fiber ring laser: II. Active mode-locking by phase modulation
} Jean Botineau, Gérard Cheval, Carlos Montes

\section{To cite this version:}

Jean Botineau, Gérard Cheval, Carlos Montes. CW-pumped polarization-maintaining Brillouin fiber ring laser: II. Active mode-locking by phase modulation. Optics Communications, 2006, 257, pp.311318. 10.1016/j.optcom.2005.07.049 . hal-00460392

\section{HAL Id: hal-00460392 https://hal.science/hal-00460392}

Submitted on 28 Feb 2010

HAL is a multi-disciplinary open access archive for the deposit and dissemination of scientific research documents, whether they are published or not. The documents may come from teaching and research institutions in France or abroad, or from public or private research centers.
L'archive ouverte pluridisciplinaire HAL, est destinée au dépôt et à la diffusion de documents scientifiques de niveau recherche, publiés ou non, émanant des établissements d'enseignement et de recherche français ou étrangers, des laboratoires publics ou privés. 


\title{
CW-pumped polarization-maintaining Brillouin fiber ring laser: \\ II. Active mode-locking by phase modulation
}

\author{
Jean Botineau, Gérard Cheval, and Carlos Montes*
}

\author{
Centre National de la Recherche Scientifique \\ Laboratoire de Physique de la Matière Condensée \\ Université de Nice - Sophia Antipolis, Parc Valrose, \\ F-06108 Nice Cedex 2, France
}

\begin{abstract}
Stimulated Brillouin backscattering in a cw-pumped long optical polarizationmaintaining fiber ring cavity excites spontaneous stable mode-locking. But the shortest pulses allowed by the Brillouin gain bandwidth imply an active modelocking, which we have achieved by using a phase modulator inside the ring. The experiment is carried out in the infrared at $1.319 \mu \mathrm{m}$, and in narrow frequency domains, close to $N$ integer multiples of the ring $F S R$, we observe two type of mode-locking: either a maximum compression of the Brillouin solitons, or a stable splitting into $N$ equally spaced pulses. Case $N=3$ is more detailed. Experiment implies a slight polarization modulation by the phase modulation. Simulations carried out through the coherent three-wave model are in very good agreement for both mode-locking mechanisms. Introduction of a small amplitude modulation together with the intra-cavity phase modulation satisfactorily explains the stable splitting domain as well as the compression domain.
\end{abstract}

published in Optics Common. 257 311-318 (2006)

*correspondig author Carlos.Montes@unice.fr 


\section{Introduction}

It is today well known that stimulated Brillouin scattering generated by a cw-pump wave inside a long optical fiber-ring resonator gives rise, under adequate conditions of finesse and pump power level, to a spontaneous mode-locked regime: the scattered wave is then self-structurated in a quasi-soliton pulse propagating back in the ring, its temporal width resulting from the competition of Stokes scattering (in the leading part of the pulse) and anti-Stokes scattering (in the rear wing) [1]. A consequence of this process is a nonlinear flight time $t_{B}$ of the pulse, where the pulse velocity is defined by the velocity of its maximum, and this flight time may be, following the conditions, greater or lower than the linear flight time $t_{r}=(F S R)^{-1}=n L / c[2][3]$.

An intriguing feature is, however, that the temporal width of the pulses obtained by this process is always much larger than what could be expected from a simple modelocking model, where the temporal width is roughly given by the inverse of the gain bandwidth. In our case, the Brillouin homogeneous gain bandwidth being $23 \mathrm{MHz}$, we may expect a pulse width shorter than 50 ns. Instead of that, the most frequent and stable trains typically correspond to an experimental width of $100 \mathrm{~ns}$, and the numerical simulations give rise, following the exact ring finesse and coupled pump power introduced in the calculation, to values between 90 and 125 ns, excepted near the nonlinear threshold or near the bifurcation towards the cw-Brillouin regime, where the pulses could be much longer [2] [3].

We must conclude that this passive mode-locking process allows the nonlinear Brillouin threshold to be reached for a small number of longitudinal modes of the fiber ring only. This is of course the case near the nonlinear threshold, where only the modes around the maximum of the gain bandwidth are excited. But when the pump input power increases (remaining however low enough to be still in the pulse domain, which corresponds in our experiment to a typical factor two for the pump power with respect to the threshold power), the nonlinear pump depletion increases along the fiber, and the number of excited longitudinal modes remains roughly the same, and therefore the Brillouin pulse width too.

However, the Brillouin gain bandwidth allows potentially a larger number of longitudinal modes to be excited, i.e. shorter pulses to be shaped. Actually, even without any 
active mode-locking device, pulses of 20 to 30ns are experimentally observed in transient regimes, typically forming trains shorter than 100 pulses. This is for example the case when the input pump wave is externally modulated by a mechanical chopper, but such narrow pulses may also occasionally be observed during the soliton regime, in particular if the fiber drum is slightly shocked. In this case, the reason seems to be an interaction between the classical stimulated Brillouin scattering and the acoustic cladding mode scattering (CBS for Cladding Brillouin Scattering or GAWBS for Guided Acoustic Wave Brillouin Scattering) [4]. Indeed, in some unstable conditions, it has been experimentally and numerically proved that Brillouin Stokes pulses may transversally excite low frequency $(\mathrm{MHz})$ acoustic waves through $\mathrm{CBS}$, which in turn may modify the pulses either by compressing them, for certain cooperative frequencies, or by widen them or strongly destabilize them for other frequencies [5]. The coupling of this Brillouin transverse resonator with the Brillouin axial resonator is generally responsible for unstable dynamics, since all kind of transverse resonances may be excited [6]. It has been shown that this effect acts through a self-phase modulation of the Brillouin pulses and is well described by a four-wave model which couples the SBS dynamics, governed by the standard resonant three-wave equations, to the CBS dynamics simulated by a forced harmonic oscillator equation [5] [7].

However, without additional forcing, this SBS-CBS coupling is too weak to ensure a permanent regime. Our aim will be to increase this coupling by an intra-cavity phase modulator forced by the solitonic pulse itself. This self-consistent interaction would be described by the four-wave model already introduced [4] [5] [7], where the transverse CBS modulation is simulated via a forced resonator coupled to the three-wave SBS system through an increased Kerr phase modulation.

In the present paper, however, we concentrate our interest on the more simple nonself-consistent problem where the intra-cavity phase modulator is activated by an external function generator emitting periodic square signals.

On one side, phase modulation of optical-fiber-guided cw signals in the presence of SBS has been considered to generate sharp pulses when the phase modulator rise time is shorter than the acoustic damping time [8] [9]. Conversion from phase modulation to amplitude modulation is a result of the transient SBS process. On other side, phase- 
modulation feedback is an effective method to achieve multiwavelength fiber ring lasers $[10]$.

In our pulsed Brillouin fiber ring laser we will use phase-modulation feedback as an active phase-locking mechanism. The function generator allows us to choose an arbitrary phase modulation frequency in the experiment, as free parameter. Moreover, we can simulate the interaction through the standard three-wave model where only the cavity reinjection contains the time dependent phase modulation. This simpler problem amazingly yields a very rich dynamics where two types of active mode-locked regimes may be observed.

\section{Experimental set-up}

Like in Part I paper [2], the experiment is carried out in the infrared at $1.319 \mu \mathrm{m}$ in a single-mode polarization-maintaining fiber inside a ring cavity. Polarization dynamics in a Brillouin fiber ring laser may lead to unstable behaviors, as has been shown for a shortlength cavity [11]. The mounting set-up (fig.1) is almost the same but with the adding of an intra-cavity phase modulator (EOSpace Inc.) near the port 3 of the circulator, where the pump as well as the scattered wave have their lowest intensity. The modulator insertion loss is $2 \mathrm{~dB}$, and exhibits an almost flat response in the range $100 \mathrm{~Hz}-100 \mathrm{kHz}$, with an attenuation of $1 \mathrm{~dB}$ at $1 \mathrm{MHz}$ and $2 \mathrm{~dB}$ at $10 \mathrm{MHz}$. This device is driven by a function generator emitting a periodic square signal, the frequency of which may be adjusted from zero to several times the Brillouin soliton roundtrip frequency $\nu_{B}=1 / t_{B}$, close to the linear roundtrip frequency $\nu_{r}=F S R=c / n L$, which in our experiment is nearby $800 \mathrm{kHz}$ (being $\nu_{B} \gtrsim \nu_{r}$ for superluminous and $\nu_{B} \lesssim \nu_{r}$ for subluminous solitons [2] [3]), and we choose an amplitude roughly equal to a phase modulation of $\pi / 3(1 \mathrm{~V})$.

\section{Experimental features}

Without phase modulation, this cw-pumped Brillouin fiber ring laser works in the pulsed regime [2], with an intensity feedback of the cavity $R \simeq 0.011$ and a Brillouin gain $G=g_{B} I_{0} L \simeq 10.5$, where $g_{B}$ is the fiber Brillouin gain coefficient, $I_{0}$ the pump intensity 
coupled in the fiber and $L=250 \mathrm{~m}$ the fiber length. The width of these Brillouin bare solitons are then approximately $100 \mathrm{~ns}$ (fig.2). When the phase modulator is turned on with an arbitrary frequency $\nu_{m}$, the Brillouin solitons are broken into unstable structures. But when $\nu_{m} \simeq N \nu_{B} \simeq N \nu_{r}$ (where $N$ is an integer), the experiment exhibits systematically two frequency domains of stable mode-locking with strongly different characteristics from the bare signal.

The first mode-locked regime is observed in a frequency domain centered around $\nu_{m}=$ $N \nu_{B}-\delta \nu_{1}$, and corresponds to an efficient compression of the Brillouin pulses (a typical width of 25-30 ns is observed, with a pulse energy equal to the bare soliton energy). A simple explanation is that, like in a classical mode-locked laser, the phase modulation coherently reinforces the lateral modes of the pulse spectrum. The frequency difference $\delta \nu_{1}$ is related to the velocity dependence of the pulses with respect to their amplitudes and their widths [2], and the mean spectral width is then $40 \mathrm{MHz}$, which fit reasonably well the gain bandwidth. The process is in this case very similar to the CBS or GAWBS effect [5], excepted the phase modulation frequency which is imposed by the function generator.

The second mode-locked regime, observed in a spectral range centered around $\nu_{m}=$ $N \nu_{B}-\delta \nu_{2}$, with $\delta \nu_{2}>\delta \nu_{1}$, was less expected. The experiment shows now a splitting of the Brillouin soliton in subpulses forming very stable trains with a repetition rate roughly equal to $N \nu_{B}$, i.e. $N$ equally spaced pulses propagating along the fiber ring instead of just one. A typical peak intensity of the subpulses is $2 / 3$ of the bare pulse peak intensity, and they are narrower, such that the whole energy is maintained. We have observed this behavior in all the frequency range allowed by our function generator, up to $N=12$. Fig. 3 shows for the case $N=3$, with the same time scale, the experimental (left) and the numerical (right) features: (a) three bare solitons obtained in the passive mode-locking regime, (b) the three narrower pulses corresponding to the first active mode-locked regime, and (c) the nine pulses corresponding to the second active mode-locked regime.

The case $N=1$ is particular because, without surprise, it exhibits no splitting domain. Note also that, in this case, the pulse compression is less pronounced. In the case $N=2$, there is a narrow overlap between the compression and the splitting domains, and we observe for these frequencies, when we turn the phase modulator on, a stable locking of the system either on the compression or on the splitting regime: it looks, at 
less experimentally, as a bistable domain. For $N>2$, the two stable domains are separated. Fig.4.a (left) shows the stable mode-locked domains for $N=1$ to 6 , with on vertical scale the pulse compression factor (with the bare soliton as reference). [The pulse compression factor is defined by the rate of the intensity pulse widths]. Blackness gives an idea of the stability of the observed structures; pale dots indicating a poor experimental reproducibility. Fig.4.b (right) gives a more detailed diagram for the case $N=3$.

In this last case, $N \nu_{B} \simeq 2400 \mathrm{kHz}$. The compression regime ranges then from 2335 to $2352 \mathrm{kHz}$, with the most stable zone near $2341 \mathrm{kHz}$, and the splitting domain from 2307 to $2326 \mathrm{kHz}$, with the best stability between 2307 and $2324 \mathrm{kHz}$. It is interesting to note that, for a well-shaped bare Brillouin soliton, the nonlinear flight time $t_{B}=\nu_{B}^{-1}$ ranges then from $1.26 \mathrm{~ns}$ (near the non linear threshold) to $1.23 \mathrm{~ns}$ (near the bifurcation towards the cw-regime), which in this case corresponds for $N \nu_{B}$ to the domain $2381-2439$ $\mathrm{kHz}$. Therefore, the modulated regimes, even the compression one, must be considered as physically different from the bare regime. This is confirmed by the case $N=1$, where the compression regime ranges from 780 to $788 \mathrm{kHz}$, whereas the bare soliton repetition frequency $\nu_{B}$ is larger than $793 \mathrm{kHz}$.

\section{Three-wave Brillouin model with modulated feedback}

In Part I [2] and former papers [12] [1] [13] [3], we have shown that the passive modelocking regime was accurately described by the 3-wave 1-D differential equation system:

$$
\begin{gathered}
\left(\partial_{t}+\frac{c}{n} \partial_{z}+\gamma_{e}\right) A_{p}=-K_{B} A_{S} A_{a}+i K_{K}\left(\left|A_{p}\right|^{2}+2\left|A_{S}\right|^{2}\right) A_{p} \\
\left(\partial_{t}-\frac{c}{n} \partial_{z}+\gamma_{e}\right) A_{S}=K_{B} A_{p} A_{a}^{*}+i K_{K}\left(2\left|A_{p}\right|^{2}+\left|A_{S}\right|^{2}\right) A_{S} \\
\left(\partial_{t}+\gamma_{a}\right) A_{a}=K_{B} A_{p} A_{S}^{*}
\end{gathered}
$$

where the pump and Stokes waves propagate along the $z$-axis of the fiber and are associated to the electric field amplitudes $A_{p}(z, t) F(x, y)$ and $A_{S}(z, t) F(x, y)$, the transverse function $F(x, y)$ standing for the transverse fiber modal profile. The Brillouin coupling constant is then :

$$
K_{B}=\sqrt{\frac{g_{B} \gamma_{a} \iint F^{4} d x d y}{4 \mu_{0} \iint F^{2} d x d y}}
$$


where $g_{B}=1.65 \times 10^{-11} \mathrm{~m} / \mathrm{W}$ is the fiber Brillouin gain constant, $\gamma_{a} \simeq 7.2 \times 10^{7} \mathrm{~s}^{-1}$ the acoustic damping constant at $13 \mathrm{GHz}$, and $\gamma_{e}$ the optical damping constant of the fiber corresponding to an attenuation of $1 \mathrm{~dB} / \mathrm{km}\left(\gamma_{e}=2.4 \times 10^{4} \mathrm{~s}^{-1}\right)$. The electrostrictive field $A_{a}(t, z)$ is proportional to the acoustic amplitude and is also depending on the fiber modal profile. Each of the two optical equations involve an optical Kerr term which acts here as a small perturbation, the coupling constant of which being given by:

$$
K_{K}=\frac{\pi n_{2} c}{n \lambda} \frac{\iint F^{4} d x d y}{\iint F^{2} d x d y}
$$

depending on the silica self-focusing coefficient $n_{2}=1.2 \times 10^{-22} \mathrm{~m}^{2} \mathrm{~V}^{-2}$, on the effective refractive index $n$ of the fiber, and on the optical wavelength $\lambda=1.319 \mu \mathrm{m}$.

Eqs.(1) must be completed by the boundary conditions:

$$
A_{p}(z=0, t)=A_{0} \quad ; \quad A_{s}(t, z=0)=\rho A_{s}(t, z=L)
$$

For the bare soliton, the reinjection coefficient is taken as a simple constant $\rho=\sqrt{R}$. In the case of the experiment presented here, $\rho$ must also describe the phase modulation versus time. Actually, as detailed in the next section, the splitting effect cannot be explained by a pure phase modulation effect for these experimental conditions. We have checked that the phase modulator did not perturb the optical intensity, but the sensitivity of the splitting regime to the polarization control in the experiment led us to suspect a slight modulation of the optical polarization by the phase modulator, which would be equivalent, for the polarization-maintaining fiber, to a weak amplitude modulation $a_{m}$. This is why we set:

$$
\rho=\left[\sqrt{R}+a_{m} f_{m}(t)\right] \exp \left[i \phi_{m} f_{m}(t)\right]
$$

where the modulation function $f_{m}$ is approximated by: 


$$
\begin{gathered}
f_{m}=1-\frac{1-\exp \left(t_{m} / 2 \tau\right)}{1-\exp \left(-t_{m} / \tau\right)} \exp \left[-\left(T-t_{0}\right) / \tau\right] ; \quad \text { for }\left(t_{0}<T<t_{0}+t_{m} / 2\right) \\
f_{m}=-\frac{1-\exp \left(t_{m} / 2 \tau\right)}{1-\exp \left(-t_{m} / \tau\right)} \exp \left[-\left(T-t_{0}\right) / \tau\right] ; \quad \text { for }\left(t_{0}+t_{m} / 2<T<t_{0}+t_{m}\right)
\end{gathered}
$$

with $T=\bmod \left(t, t_{m}\right), \tau / t_{m}=4.24 \times 10^{-4}$, and $\phi_{m}=0.951997$ according to the experimental relaxation time $\tau$ and phase modulation amplitude. The modulation frequency is then $\nu_{m}=1 / t_{m}$, but for the calculations we use as control frequency parameter the dimensionless term $\Omega=\nu_{m} / \nu_{r}=\omega t_{r} / 2 \pi$, where $t_{r}=n L / c$ is the ring linear flight time.

\section{Numerical agreement with the experiment}

In the model presented in former section, the parameter $a_{m}$, which describes the parasitic polarization modulation of light by the phase modulator, must be adjusted to fit the experimental results. We have chosen to perform the numerical study for the 3rd mode, and that in two cases:

(1) the actual experimental conditions, with a mean intensity feedback $\langle R\rangle=0.0115$ and an input pump power of $60 \mathrm{~mW}(G=10.5)$, corresponding to the spontaneous pulsed regime in the absence of phase modulation,

(2) to conditions belonging to the cw-Brillouin regime without phase modulation, with $\langle R\rangle=0.00823$ and an input pump power of $80 \mathrm{~mW}(G=14)$, whose experimental study is not presented here, and corresponds to case (4) (table II) of Part I paper [2].

In both cases, we obtain, within finite frequency bands, the two mode-locked structures described above experimentally. There is however an important physical difference between the two cases: in case $(2)$, no amplitude modulation $\left(a_{m}=0\right)$ is required to get the splitting regime, whereas in case (1) we must take $a_{m}=6 \times 10^{-3}$ to fit the experiment. We show on Fig.3 (right) the numerical results in order to make easier comparison with experimental features, and on figure 5 the numerical graph corresponding to the experimental results for the 3rd mode plotted on Fig.4 (right). Finally we plot on figure 6 the stable 3 -times splitting regime corresponding to case (2). 


\section{Conclusion}

Phase modulation in a cw-pumped single-transverse mode long fiber ring cavity exhibits two types of active mode-locking allowing to draw benefit from the full spectral width of the Brillouin gain. The first one corresponds to the compression of Brillouin solitons obtained through passive mode-locking in the case of stationary reinjection in the ring. The second one implies a weak polarization modulation to be added to the phase modulation, at least in the pulsed zone of the Brillouin ring, which is consistent with the experimental behavior.

The very good agreement between experiment and numerical simulations confirms the universal validity of the 3-wave 1-D model we use to describe this system. The model predicts also the two same mode-locked regimes, even without light-polarization modulation in the cw-zone of the passive ring configuration.

If the phase modulation signal was induced by the Stokes pulses, such a configuration would fit very closely the CBS or GAWBS process in the case of a strong coupling of the cladding acoustic modes with the Brillouin pulses. This self-consistent problem will be the purpose of a next experiment. 


\section{References}

[1] E. Picholle, C. Montes, C. Leycuras, O. Legrand, and J. Botineau, Observation of dissipative superluminous solitons in a Brillouin fiber ring laser, Phys. Rev. Lett. 66, 1454-1457 (1991).

[2] J. Botineau, G. Cheval, and C. Montes, CW-pumped polarization-maintaining Brillouin fiber ring laser : I. Self-struturation of Brillouin solitons.

[3] C. Montes, D. Bahloul, I. Bongrand, J. Botineau, G. Cheval, A. Mamhoud, E. Picholle, and A. Picozzi, Self-pulsing and dynamic bistability in cw-pumped Brillouin fiber ring lasers, J. Opt. Soc. Am. B 16, 932 (1999) and references therein.

[4] C. Montes, I. Bongrand, E. Picholle, J. Botineau, A. Picozzi, and G. Cheval, Soliton compression and locking in a Brillouin fiber ring laser, in Optical Pulse and Beam Propagation III, Yehuda B. Band, Editor, Proceedings of SPIE Vol. 4271 (2001).

[5] I. Bongrand, C. Montes, E. Picholle, J. Botineau, A. Picozzi, G. Cheval, and D. Bahloul, Soliton compression in Brillouin fiber lasers, Opt. Lett. 19, 1475 (2001).

[6] E. Picholle and A. Picozzi, Guided-acoustic-wave resonances in the dynamics of a stimulated Brillouin fiber ring laser, Opt. Commun. 135 327-330 (1997).

[7] I. Bongrand, E. Picholle, and C. Montes, Coupled longitudinal and transverse stimulated Brillouin scattering in single-mode fibers, Eur. Phys. J. D 20, 121-127 (2002).

[8] A. Höök, Pulse formation in phase-modulated optical fiber signals due to stimulated Brillouin scattering J. Opt. Soc. Am. B 8, 1284 (1991).

[9] G. Grosso and A. Höök, Generation of short pulses by stimulated Brillouin scattering in optical fibers, J. Opt. Soc. Am. B 10, 946 (1993). 
[10] K. Zhou, D. Zhou, F. Dong, and N.Q. Ngo, Room-temperature multiwavelength erbium-doped fiber ring laser employing sinusoidal phase-modulation feedback, Opt. Lett. 28, 89392003$)$.

[11] S. Randoux and J. Zemmouri, Polarization dynamics of a Brillouin fiber ring laser, Phys. Rev A 59, 1644 (1999).

[12] J. Botineau, C. Leycuras, C. Montes, and E. Picholle, Stabilization of a stimulated Brillouin fiber ring laser by strong pump modulation, J. Opt. Soc. Am. B 6, 300-312 (1989).

[13] C. Montes, A. Mamhoud, and E. Picholle, Bifurcation in a cw-pumped Brillouin fiber-ring laser: coherent soliton morphogenesis, Phys. Rev. A 49, 1344 (1994). 


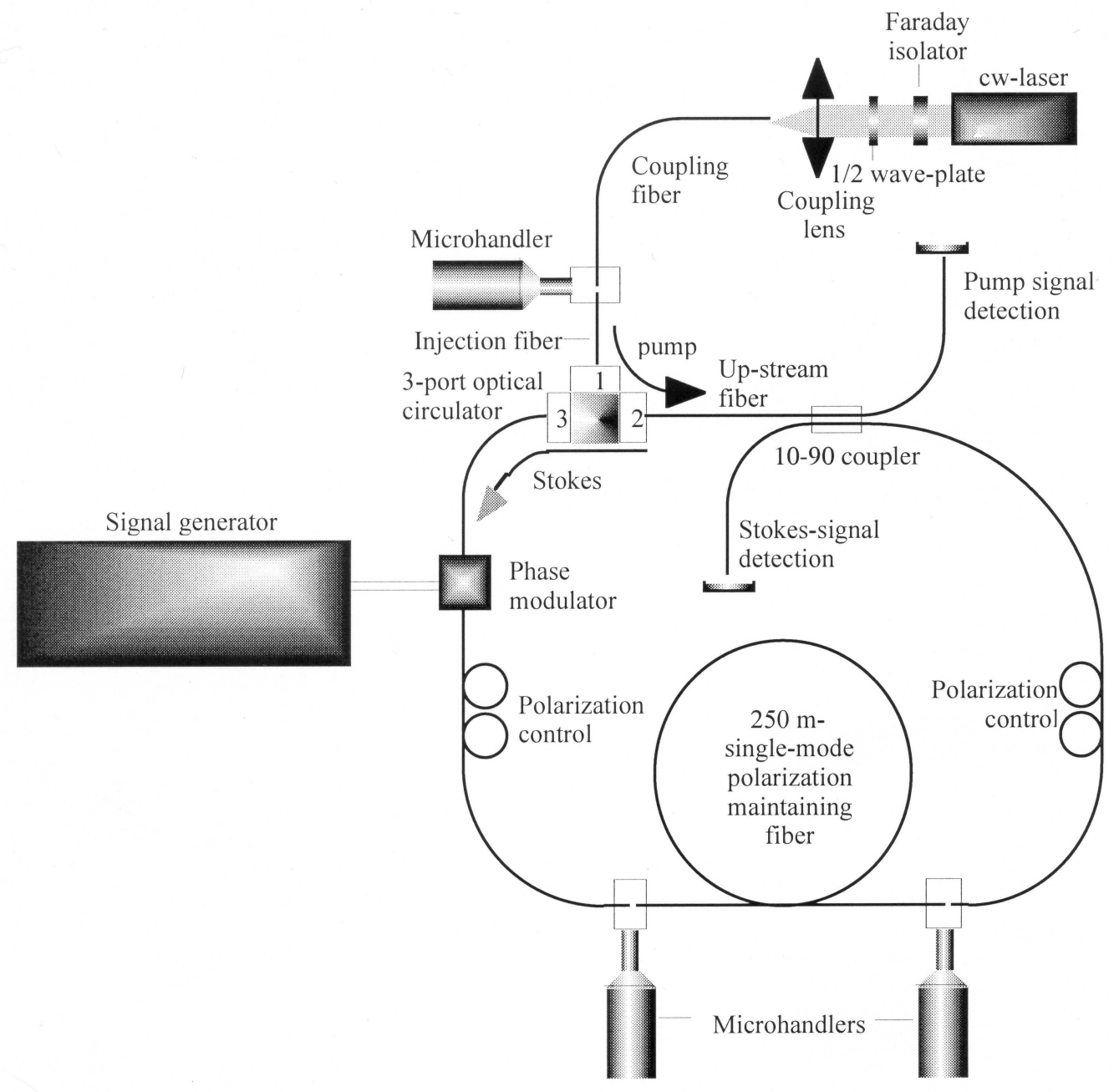

Figure 1: Experimental set-up: same mounting set as in figure 1 of Part I paper [2] with additional insertion of the intra-cavity phase modulator. 


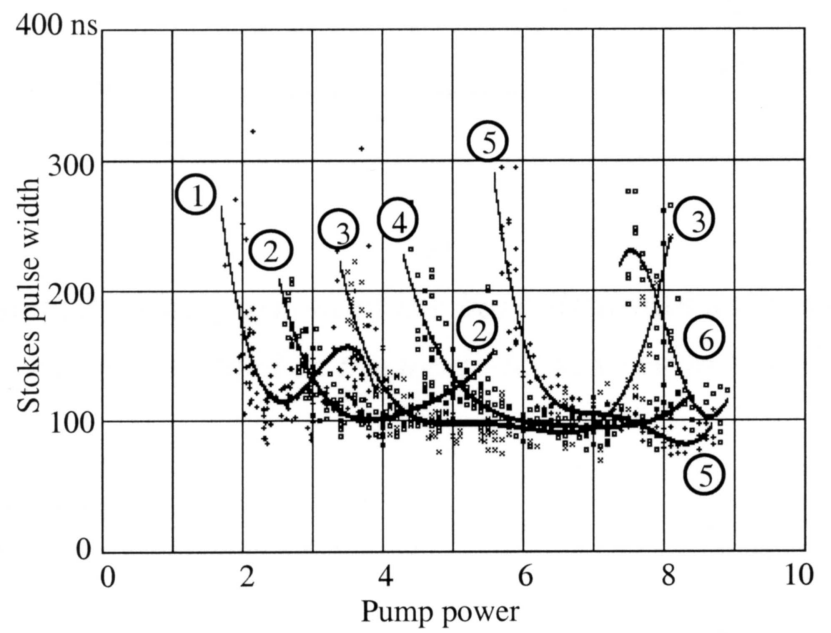

Figure 2: Brillouin pulse width vs. pump power for different values of $R$ in the passive fiber ring configuration. 

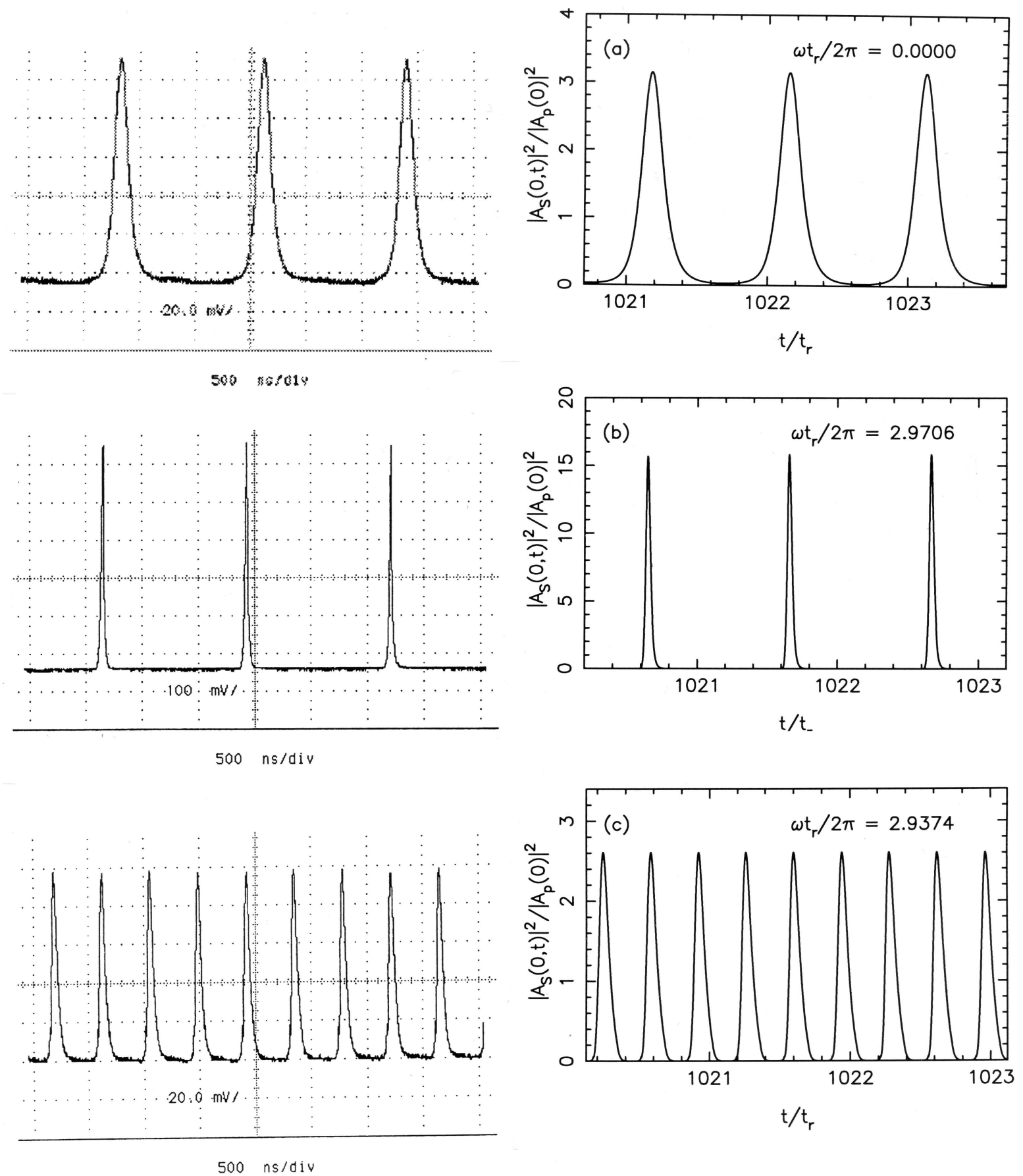

Figure 3: Trains of Brillouin pulses: experimental (left), numerical (right). (a) Unperturbed Brillouin solitons for $P=60 \mathrm{~mW}$ and $R \simeq 1 \%$; (b) train of three compressed pulses (by a factor 5 ) trough intracavity phase modulation at $\Omega=\omega t_{r} / 2 \pi=2.9706 \Rightarrow 2342 \mathrm{kHz}$; (c) three times splitted pulses (by the 3rd mode) trough intracavity phase modulation at $\Omega=\omega t_{r} / 2 \pi=2.9374 \Rightarrow 2315 \mathrm{kHz}$. 


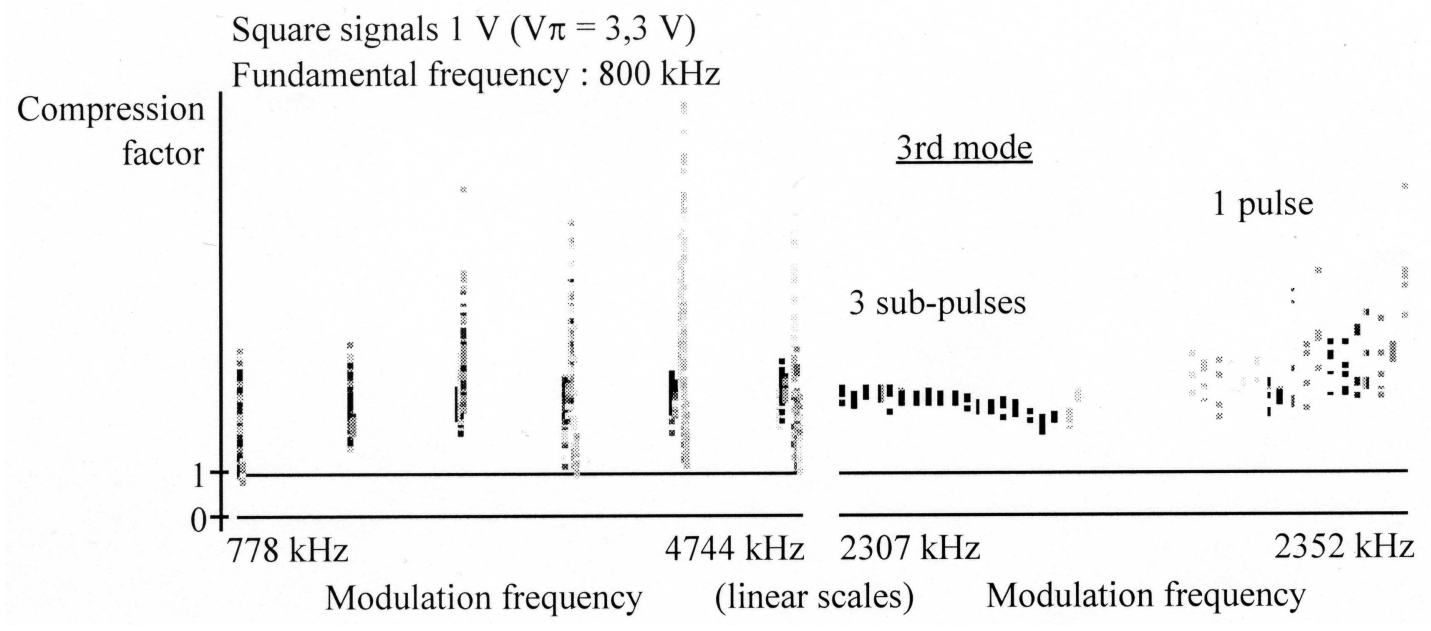

Figure 4: Experimental recording: compression factor vs. modulation frequency showing the frequency zones of mode-locking by phase modulation. Left figure: the six first modes showing the doublets of splitting domains (left) and compression domains (right); for the two first modes these domains nearly overlap. Right figure: zoom for the 3rd mode domain. Blackness indicates better stability. The frequency values correspond to the extrema of the graphs. 
mode 3: $\quad G=10.5 ; \quad\langle R>=0.0115$

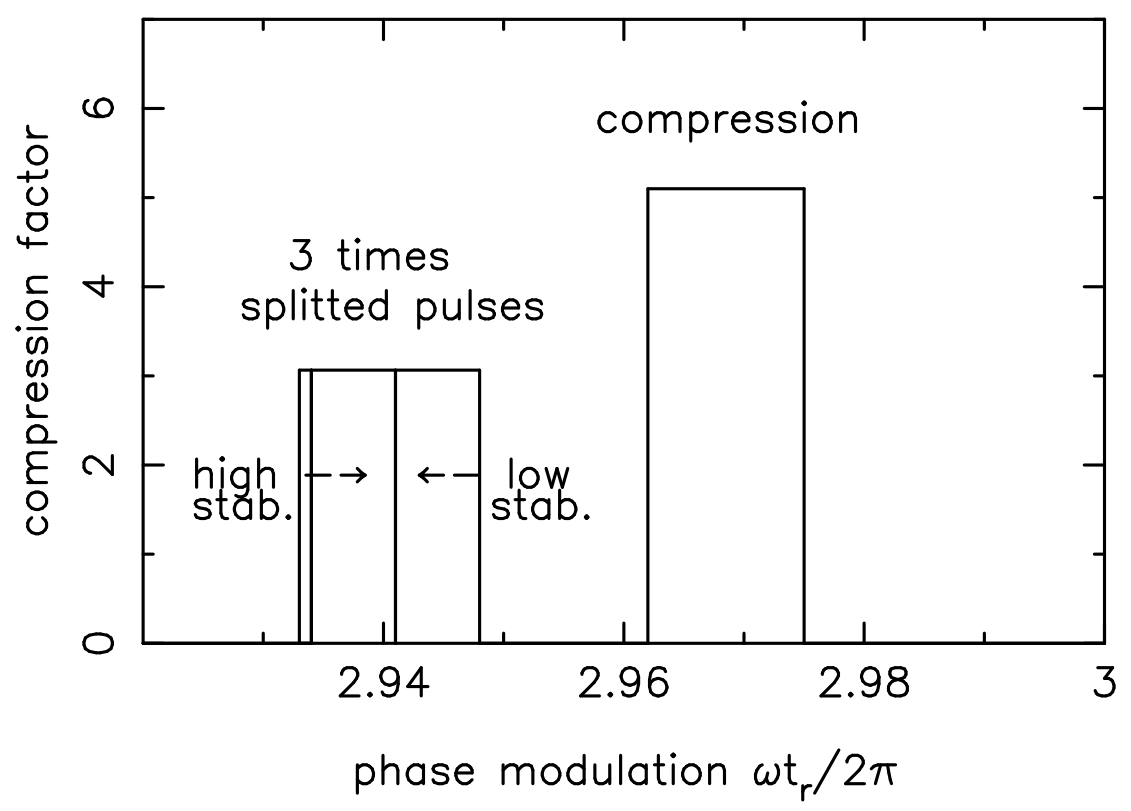

Figure 5: Numerical 3rd mode frequency bands: compression factor vs. phase modulation frequency for both stable frequency domains. Left frequency band corresponds to the 3times splitted Brillouin pulses showing high and low stability domains. Right frequency band corresponds to the 5-times single compression domain. 


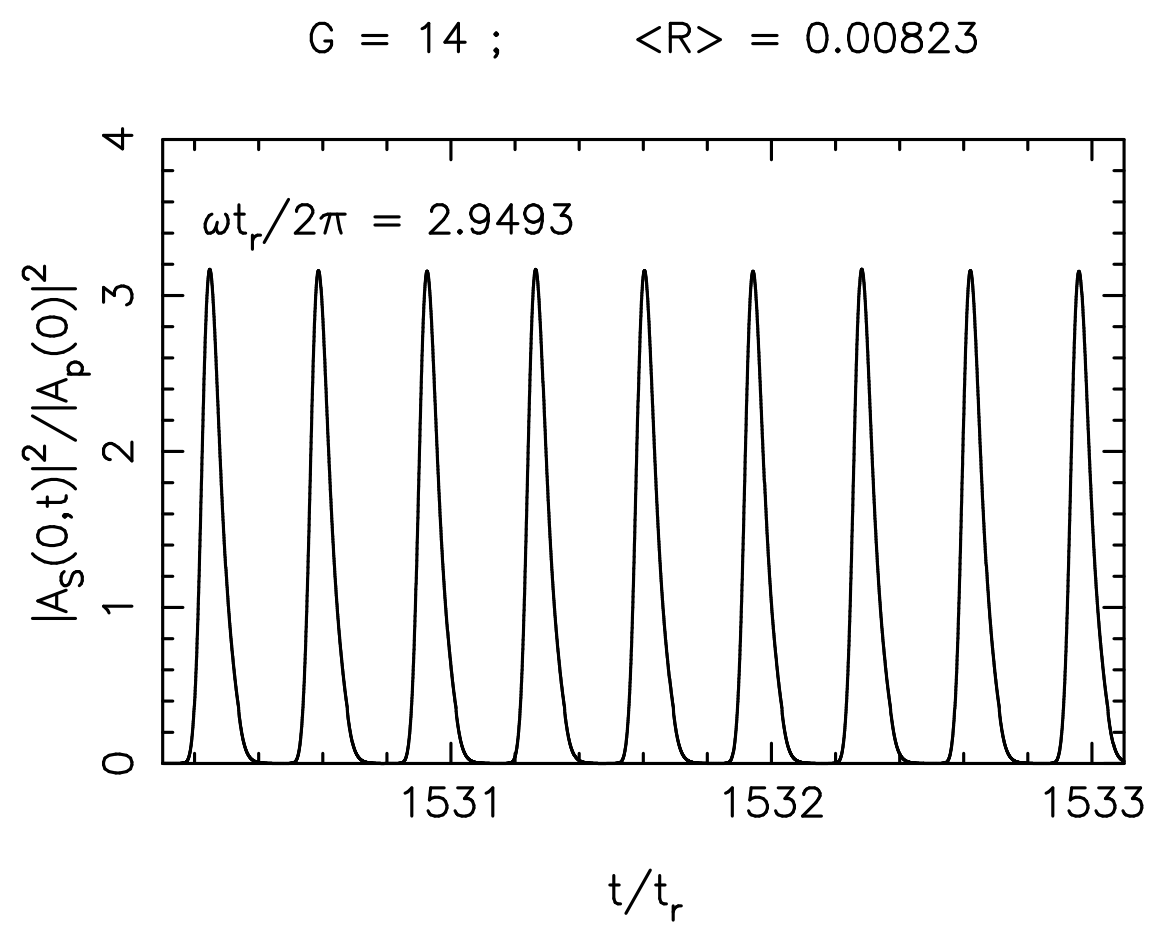

Figure 6: Numerical train of 3-times splitted Brillouin pulses excited by intracavity phase modulation $\left(a_{m}=0\right)$ for the mode-locking case (2). In the absence of modulation it corresponds to case (4) of Part I paper [2] with $G=14$ and $R=0.00823$. Here, intracavity (3rd mode) phase modulation is at $\Omega=\omega t_{r} / 2 \pi=2.9493$. 